\title{
SEROLOGY OF THE SYSTEMIC LUPUS ERYTHEMATOSUS IN EGYPTIAN PATIENTS
}

By

ALYAA A. FARID ${ }^{1}$, RWAN A. EISSA ${ }^{2}$, AHMED M. NADA ${ }^{2}$, MONA M. ELAMIR ${ }^{3}$, And AZZA M. ELAMIR ${ }^{1}$

Faculty of Science, Cairo University ${ }^{1,}$ Giza and Faculty of Biotechnology ${ }^{2}$, October University for Modern Sciences and Arts (MSA), $6^{\text {th }}$ October, Egypt

( ${ }^{*}$ Correspondence: alyaafarid@yahoo.com)

\section{Abstract}

Systemic lupus erythematosus (SLE) is a prototypic multisystem autoimmune disease with a vast spectrum of clinical presentations including almost all body organs and tissues. The incidence of lupus has tripled in the last 40 years, mainly due to the improved diagnosis. For more than 60 years the presence of autoantibodies in patients with SLE has been known. A great effort is being made for understanding the diagnostic, pathogenic and prognostic meaning of these autoantibodies. The study demonstrated the significance of autoantibodies test in Egyptian SLE patients. The results showed that 93.3\% of patients have positive ANA test, $90 \%$ have positive anti-dsDNA. Anti-Sm antibodies were in $40 \%$ and $49.3 \%$ of patients have positive anti-nucleosome antibody test. Also, 30.6\% of patients deve-loped a positive anti-histone test \& $26 \%$ have positive anti-ribosomal test. Anti-phospholipids antibodies were found in $44.6 \%$ of patients. Levels of C3 \& C4 $(0.552 \pm 0.43 \& 0.125 \pm 0.12$, respectively) were lower than that of control group ( $1.2 \pm 0.35 \& 0.42 \pm 0.54$ for C3 \& C4, respectively).

Key words: Egypt, Systemic lupus erythematosus, Autoantibodies test

\section{Introduction}

Systemic lupus erythematosus (SLE) is an autoimmune disease characterized by autoreactive $B$ and $T$ cells and production of a broad and heterogeneous group of autoantibodies (Cozzani et al, 2014).

Antibodies that react with self-molecules occur in healthy individuals and are referred to as the natural antibodies or autoantibodies (Casali and Schettino, 1996). These selfantigens may be present in all types of cells or extensively specific for a specific cell type in one organ of the body. It may include proteins, nucleic acids, carbohydrates, lipids or various combinations of these. In systemic lupus, the dominant antigens are the ribonucleo-proteins (RNPs) or deoxyribonucleoproteins (Elkon and Casali, 2008).

Hargraves et al. (1948) demonstrated the presence of autoantibodies in SLE when they described lupus phenomenon. This was proven when it was understood that lupus was due to neutrophil phagocytosis of cell nuclei opsonized by autoantibodies. DNA antibodies were identified (Ceppellini et al, 1957) and Tan and Kunkel (1966) discover ed autoantibodies directed to antigens differ- ent from DNA and described the anti-Sm antibodies. These autoantibodies were used as useful biomarkers for SLE disease, and gave information about basic mechanisms of the loss of tolerance and inflammation (Elkon and Casali, 2008). Sherer et al. (2004) reported that 116 autoantibodies were identified in patients with SLE. In SLE, especially in the systemic form, auto-antibodies directed to the nuclear (ANAs), cytoplasmtic, and cellular membrane antigens are considered the serologic hallmark. ANAs consisted of various types of auto-antibodies characterized by the different antigen specificities. The nuclear antigens include the single strand (SS) and double strand (DS) DNA, histone proteins, nucleosome, centromere proteins, and extractable nuclear antigens (Smith antigen $(\mathrm{Sm})$, Ro, La, ribonucleoprotein (RNP)...etc.). ANAs are present in about $95 \%$ of SLE patients with an active disease (Egner, 2000). In the patients with prevalent cutaneous lesions, ANAs have been found positive in $75 \%$ of cases. Hamdy et al. (2010) in Egypt, reported that most of SLE studied patients were characterized predominantly by the muco-cutaneous and the 
hematological manifestations, and the QoL of SLE patients with renal involvement as measured by SF-36 was poor than that of the healthy control in all the domains except emotional limitations.

The present study aimed to highlight the most promising and significant ones from the immuno-pathological and clinical perspectives point of view.

\section{Patients and Methods}

The study involved 150 patients with SLE attending the Department of Rheumatology and Rehabilitation, Cairo University Hospitals. All patients fulfilled the 1997 American College of Rheumatology criteria for SLE (Hochberg, 1997) and their disease activity was assessed by SLE Disease Activity Index or SLEDAI (Bombardier et al, 1992).

Qualitative analysis of antinuclear autoantibodies was done using anti-nuclear antibody immunoblot kit (Germany). The antidouble-stranded deoxyribonucleic acid antibody, anti-phospholipid antibodies, i.e., IgM \& IgG anti-cardiolipin antibody, and IgM \& IgG anti- $\beta 2$ glycoprotein I antibody were measured using commercial ELISA kits and the omplement components C3 \& C4 were measured by Nephelometry (Germany). The signed informed consent was obtained from all participants and the study was approved by the institute's Ethics Committee.

\section{Results}

The study included 150 patients with SLE (136 females \& 14 males), whereas the apparent healthy controls were 150 individuals (130 females \& 20 males) cross matched and ethnic background. Patients and controls were $30.8 \pm 5.6 \& 29 \pm 6.5$ years respectively. In SLE patients, disease onset was $29.3 \pm 6.8$ years and duration was $12.5 \pm 1.2$ years. The mean SLEDAI score was 13.9 \pm 9.6 , indicated that all patients had active disease. The clinical pictures were hematological involvement (52\%), lupus nephritis (52\%), inflammatory arthritis $(67 \%)$, and secondary antiphospholipid antibody syndrome (12\%). The complement components C3 \& C4 were low in $39 \%$ and $31 \%$ of patients, respectively.

The autoantibody profile of patients $\mathrm{C} 3 \&$ C4 mean levels were summarized (Tab.1).

Table 1: Serology and complement C3 \& C4 in SLE patients.

\begin{tabular}{|l|c|c|}
\hline \multicolumn{1}{|c|}{ Autoantibody profiles } & Number of patients $(\%)$ & No of controls $(\%)$ \\
\hline ANA & $140(93.3 \%)$ & $5(3.3 \%)$ \\
\hline Anti-dsDNA & $135(90 \%)$ & $8(0.53 \%)$ \\
\hline Anti-nucleosome & $74(49.3 \%)$ & $2(1.3) \%$ \\
\hline APLA $(\beta 2 \mathrm{GP} 1, \mathrm{LAC})$ & $67(44.6 \%)$ & $1(0.6 \%)$ \\
\hline Anti-sm & $60(40 \%)$ & $2(1.3 \%)$ \\
\hline Anti-histone & $46(30.6 \%)$ & $1(0.6 \%)$ \\
\hline Anti-ribosomal & $39(26 \%)$ & $3(2 \%)$ \\
\hline C3 (g/L) & $0.552 \pm 0.43$ & $1.2 \pm 0.35$ \\
\hline C4 $(\mathrm{g} / \mathrm{L})$ & $0.125 \pm 0.12$ & $0.42 \pm 0.54$ \\
\hline
\end{tabular}

ANA: Anti-Nuclear Antibody, Anti-dsDNA: double strand (ds) DNA, APLA: antiphospholipids antibodies, antism: anti- smith antibody, C3 and C4: complement proteins.

\section{Discussion}

Generally speaking, the systemic lupus erythematosus (SLE) is the chronic autoimmune disease affecting almost all organ systems, characterized by exacerbations (or flares) of the disease activity and the disease damages (Müller and Muir, 2014). The measures of disease activity include; SLE disease activity index, and British Isles Lupus Assessment group or BILAG disease activity index (Stoll et al, 1996) and SLE activity Measure or SLAM (Bae et al, 2000). Altho- ugh 180 or more self-antigens were reported as targets in SLE yet, few were common, this subset, which includes the anti-Sm/ RNP, anti-Ro/SS-A, anti-La/SS-B, anti-DS/ DNA, and several others, consists primarily of the nucleic acid (DNA or RNA) associated proteins (Han et al, 2015).

In the present study, $93.3 \%$ of the patients gave positive ANA test; and $90 \%$ of them exhibited positive anti-DS/DNA. ANA are autoantibodies to the cells nuclei, $98 \%$ of all patients had a positive ANA test, making it 
the most sensitive diagnostic test for SLE (Walravens, 1987). The ANAs were present in $5-10 \%$ of controls and people with other diseases; such as the rheumatoid arthritis (Grygiel-Górniak et al, 2018). Also, 20\% of healthy women had a weakly positive ANA, and majority never developed lupus signs.

Anti-DS/DNA is a specific type of ANA antibody found in about $30 \%$ of SLE patients. Less than $1 \%$ of healthy individuals have Anti- DS/DNA, making it a helpful marker for confirming SLE. The presence of anti- DS/DNA antibodies often suggested more serious lupus, such as lupus nephritis. High amounts of anti-DNA antibodies were present in active lupus nephritis.

On the other hand, the anti-Sm antibodies were in $40 \%$ of patients. Anti-Sm is an antibody to a ribonucleoprotein found in the cell nucleus. It is found exclusively in people with lupus (Kurien and Scofield, 2006). It is present in $20 \%$ of patients with the disease, less than $1 \%$ of healthy individuals and rarely found in people with other rheumatic diseases. It proved helpful in diagnosis of systemic lupus. Unlike anti-DS/DNA, antiSm did not correlate with the presence of lupus nephritis.

In the present study, $49.3 \%$ of patients have positive anti-nucleosome antibody test. The first serological marker of the SLE described was anti-nucleosome antibodies. Nucleosomes are considered as the major autoantigen in SLE, played an important pathogenetic role and about $85 \%$ of patients have positive test (Bruns et al, 2000). Also, van der Vlag and Berden (2011) reported that nucleosome antibodies possess the important role in the pathogenesis of SLE, as being the first antibodies to appear in murine lupus models before the onset of other autoantibodies.

In the present study, $30.6 \%$ of the patients developed a positive anti-histone test. Antihistone is an antibodies to histones, proteins that help to lend structure to DNA. It is usually found in both people with druginduced lupus and people with systemic lupus (Cozzani et al, 2014). However, it was not specific enough to systemic lupus to be used as a diagnostic marker. But, $26 \%$ of patients have positive anti-ribosomal test. Nagai et al. (2005) reported that autoantibodies to ribosomal proteins were detected in $12-16 \%$ of SLE patients and have been associated with some disease manifestations, including the lupus psychosis and hepatitis.

In the present study, anti-phospholipids antibodies were found in $44.6 \%$ of patients, which were not confined to SLE patients only; but it was found in other autoimmune diseases, infections, malignant, and druginduced disorders as well as in some apparently healthy individuals. In addition, Chu et al. (1988) reported that anti-phospholipids antibodies are positive in $30-40 \%$ of SLE patients. They added that only $1 / 3$ of them developed clinical features of anti-PL syndromes such as the venous thrombosis, arterial thrombosis, recurrent pregnancy loss, hemolytic anemia and skin ulcers (Chu et al, 1988).

The present study reported $0.552 \pm 0.43 \&$ $0.125 \pm 0.12$ were for C3 \& C4, respectively. These levels were lower than that of control group $(1.2 \pm 0.35 \& 0.42 \pm 0.54$ for C3 \& C4, respectively). The serum complement test measured the proteins consumed levels during the inflammatory process (Perrin et al, 1973). So, low complement levels reflect inflammation within the body. One of the diagnostic hallmarks of lupus is low C3 \& C4 levels. Low C3 only is not specific to lupus; but combined low C3 \& C4 is usually seen in lupus. Therefore, the patient's levels of C3 \& C4 indicated the activity of the disease. Where, low levels of $\mathrm{C} 3$ and $\mathrm{C} 4$ suggested active disease and a current flare or a flare is imminent. Normal levels of C3 and $\mathrm{C} 4$ indicated that the disease is more likely calm; but it does not mean that the patient would not experience a flare. The consistently low level of C3 indicated the possibility of developing lupus nephritis in the future. In this case, the doctor must watch the kidneys and ask the patient to 
return every two/three months for urine analysis (Walport, 2002).

\section{Conclusion}

Lupus occurs if immune system attacks healthy tissue (autoimmune disease). People with an inherited predisposition for lupus, when come into contact with environmental trigger lupus.

\section{References}

Bae, SC, Koh, HK, Chang, D, Kim, M, Park, J, et al, 2000: Reliability and validity of systemic lupus activity measure-revised (SLAM-R) for measuring clinical disease activity in systemic lupus erythematosus. Lupus 10, 6:405-9.

Bombardier, C, Gladman, DD, Urowitz, MB, Caron, D, Chang, CH, 1992: Derivation of the SLEDAI: A disease activity index for lupus patients. The committee on prognosis studies in SLE. Arthritis Rheum. 35:630-40.

Bruns, A, Blass, S, Hausdorf, G, Burmester, GR, Hiepe, F, 2000: Nucleosomes are major $T$ and $\mathrm{B}$ cell autoantigens in the systemic lupus erythematosus. Arthritis Rheumat. 43: 2307-15.

Casali, P, Schettino, EW, 1996: Structure and function of natural antibodies. Curr. Top. Microbiol. Immunol. 210:167-79

Ceppellini, P, Polli, E, Celada, F, 1957: A DNA-reacting factor in serum of a patient with lupus erythematous diffusus. Proceed. Soc. Exp. Biol. Med. 96:572-4.

Chu, P, Pendry, K, Blecher, TE, 1988: Detection of lupus anticoagulant in patients' attending an anticoagulation clinic. Brit. Med. J. 297: 1449-53

Cozzani, E, Drosera, M, Gasparini, G, Parodi, A, 2014: The serology of lupus erythematosus: Correlation between immunopathological features and clinical aspects. Autoimm. Dis. 4:1-13.

Egner, W, 2000: The use of laboratory tests in diagnosis of SLE. J. Clin. Pathol. 53, 6:424-32

Elkon, K, Casali, P, 2008: Nature and functions of autoantibodies. Nat. Clin. Pract. Rheumatol. 4, 9: 491-8.

Grygiel-Górniak, B, Rogacka, N, Puszczewi cz, M, 2018: Antinuclear antibodies in healthy people and non-rheumatic diseases - diagnostic and clinical implications. Reumatol. 56, 4:243-8.

Hamdy, S, Gamal, T. Khalil, AK, Ibrahim, NI, 2010: Pattern of systemic lupus erythematosus in Egyptian patients: the impact of disease activity on the quality of life. Pan Afr. Med. J. 6:14-20.
Han, S, Zhuang, H, Shumyak, S, Yang, L, Reeves, WH, 2015: Mechanisms of autoantibody production in systemic lupus erythematosus. Front Immunol. 6:22-8.

Hargraves, M, Richmond, H, Morton, R, 1948: Presentation of two bone marrow components, the tart cell and the LE cell. Mayo Clin. Proceed. 21:25-8.

Hochberg, MC, 1997: Updating the American College of Rheumatology revised criteria for the classification of systemic lupus erythematosus. Arthritis Rheum. 40:1725

Kurien, BT, Scofield, RH, 2006: Autoantibody determination in the diagnosis of systemic lupus erythematosus. Scand. J. Immunol. 64: 227-35.

Müller, MM, Muir, TW, 2014: Histones: At the crossroads of peptide and protein chemistry. Chem. Rev. 115, 6: 2296-349.

Nagai, T, Arinuma, Y, Yanagida, T, Yamamoto, K, Hirohata, S, 2005: Anti-ribosomal P protein antibody in human systemic lupus erythematosus up-regulates the expression of proinflammatory cytokines by human peripheral blood monocytes. Arthritis Rheum. 52, 3:847-55.

Perrin, LH, Lambert, PH, Nydegger, UE, Miescher, PA, 1973: Quantitation of C3PA (properdin factor B) and other complement components in diseases associated with a low C3 level. N. Engl. J. Med. 2:16-27.

Sherer, Y, Gorstein, A, Fritzler, MJ, Shoenfeld, Y, 2004: Autoantibody explosion in systemic lupus erythematosus: more than 100 different antibodies found in SLE patients. Semin. Arthritis Rheum. 34: 501-37.

Stoll, TS, Stucki, G, Malik, J, 1996: Further validation of the BILAG disease activity index in patients with SLE. Ann. Rheum. Dis. 55, 10:756-60.

Tan, EM, Kunkel, HG, 1966: Characteristics of a soluble nuclear antigen precipitating with sera of patients with systemic lupus erythematosus. J. Immunol. 96:464-71.

van der Vlag, J, Berden, JHM, 2011: Lupus nephritis: Role of anti-nucleosome antibodies. Semin. Nephrol. 31:376-89.

Walport, MJ, 2002: Complement and systemic lupus erythematosus. Arthritis Res. 4: S279-93.

Walravens, M, 1987: Systemic diseases and the detection of nuclear and anticytoplasmic antibodies: A historical review. Clin. Rheumatol. 6:9-17. 